\title{
Las traducciones al castellano de los libros de Keynes
}

\author{
ROCIO SANCHEZ-LISSEN \\ Universidad de Sevilla, Facultad de Ciencias Económicas y Empresariales, Avda. Ramón y Cajal, 1, \\ 41018 Sevilla, España. E-mail : rslissen@us.es
}

\begin{abstract}
RESUMEN
Este artículo se centra en las traducciones al castellano de seis libros de John Maynard Keynes, así como en las principales referencias a España halladas en ellos, estructurándose en dos partes. La primera ofrece una breve biografía de Keynes relevante para este trabajo, y la segunda presenta por orden cronológico de su publicación original en inglés los libros de Keynes traducidos al castellano. De esta manera, las referencias a esos libros traducidos comienzan con The Economic Consequences of the Peace (1919), editado en España en 1920 y terminan con The General Theory of Employment, Interest and Money (1936), editado en Méjico en 1943. Se ofrece también un anexo con la relación de libros traducidos y sus posteriores ediciones.
\end{abstract}

Palabras clave: Keynes, España, libros traducidos.

\section{The Translations into Spanish of the Keynes' Books}

\begin{abstract}
This article focuses on the translations into Spanish of six books of John Maynard Keynes, as well as in the major references to Spain found in them. It has been structured in two parts. The first offers a brief biography of Keynes relevant to this work, and the second presents in chronological order of its original publication in English, the books of Keynes translated into Spanish. In this way, the references to these translated books begin with The Economic Consequences of the Peace (1919), published in Spain in 1920 and end with The General Theory of Employment, Interest and Money (1936), book published in Mexico in 1943. It is also an annex with the relationship of translated books and its subsequent editions.
\end{abstract}

Keywords: Keynes, Spain, Translated Books.

Clasificación JEL: B22, B31, E12

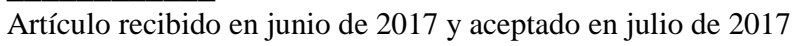

Artículo disponible en versión electrónica en la página www.revista-eea.net, ref. ə-35304 


\section{INTRODUCCIÓN}

Tanto la vida como la obra de Keynes han sido objeto de numerosos trabajos publicados en España. En este sentido cabe señalar la biografía sobre Keynes de Roy Harrod (1951), publicada en castellano en 1958 con el título La vida de John Maynard Keynes o la más reciente de Robert Skidelsky aparecida en 2013 y titulada John Maynard Keynes. Respecto a la obra de Keynes, entre los principales autores españoles especialistas en ella, cabe mencionar a Enrique Fuentes Quintana (1981, 1983, 1988), Luis Ángel Rojo (1965, 1984, 2012), Antonio Torrero (1998, 2009, 2010, 2013), y Juan Velarde (1983, 1988).

Concretamente este artículo se ocupa de los seis libros de Keynes que han sido traducidos al castellano, así como de las principales referencias a España que hay en ellos. Para llevar a cabo nuestro propósito dividimos este trabajo en dos partes: la primera aporta unas notas biográficas sobre Keynes relevantes para este estudio, y la segunda se centra en esas referencias que hace a España en sus libros, tomando como punto de partida los 29 volúmenes de The Collected Writings de Keynes, editados por MacMillan y Cambridge University Press entre 1971 y 1989. Se ha elegido el criterio del año de publicación de la versión original de las obras de Keynes para exponer las ideas en la segunda parte de este trabajo. En el anexo se presenta la relación de libros de Keynes traducidos al castellano, así como sus posteriores ediciones.

\section{NOTAS BIOGRÁFICAS DE JOHN MAYNARD KEYNES}

John Maynard Keynes nació en Cambridge el 5 de junio de 1883. Su padre, John Neville Keynes, filósofo y economista, publicó el conocido tratado Scope and Method of Political Economy, y su madre, Florence Ada Brown, fue la primera mujer alcaldesa de Cambridge. No se puede entender a Keynes sin la influencia, en cuanto a formación se refiere, recibida de sus padres. En este sentido, según Fuentes Quintana ${ }^{1}$ existen tres claves para poder interpretar a Keynes: su familia, su formación en Cambridge y los problemas de orden político, social y económico que él vivió en Inglaterra. La sólida formación inicial recibida por Keynes en Eton, donde ingresó con 14 años, consistió en estudiar matemáticas, literatura, historia y los clásicos.

En 1902 pasó al King’s College de Cambridge, en el que permaneció hasta 1906 y donde se integraría en el llamado Grupo de Bloomsbury, formado entre otros por Leonard Woolf, Lytton Strachey y Clive Bell. Robert Skidelsky (2013) ha reconocido que dos figuras clave para Keynes en el King's fueron, de un lado, Oscar Browning, cuya influencia le llevó a profundizar en el estudio de la historia y la política, rechazando la tendencia a la especialización por

${ }^{1}$ (1983), p. 246 
considerarla perturbadora, y de otro G. L. Dickinson, quien le transmitió su filosofía idealista por el método de diálogos socráticos.

Keynes se graduó en Matemáticas con 22 años, en 1905, con el puesto número 12 entre todos los alumnos de Cambridge, y durante el siguiente curso estudió Economía con Marshall y Pigou. En 1906 abandonó la Universidad para preparar unas oposiciones a la Administración Pública, consiguiendo el número dos, y fue destinado a servir en la Oficina de la India, aunque no llegó a visitar ese país. Fruto de esa experiencia laboral fue la publicación en 1913 de su primer libro Indian currency and Finance. En 1909 dejó la Administración Pública para pasar al King's College como Lector de Economía. Dos años después fue nombrado editor del Economic Journal y secretario de la Royal Economic Society.

Tras la primera guerra mundial, Keynes mostró su amplio desacuerdo con las condiciones impuestas por el Tratado de Versalles a las naciones vencidas, especialmente Alemania, lo cual le llevó a preparar en 1919 su excepcional libro The Economic Consequences of the Peace, que apareció en las librerías en diciembre de ese año. Tres años después, en 1922, publicaría A revision of the Treaty.

A partir de 1919, Keynes vuelve a Cambridge y en 1921 publica el libro A Treatise on Probability. Al año siguiente de esta publicación, y con objeto de influir sobre la reconstrucción de Europa tras la Gran Guerra, comenzaron sus colaboraciones en el Manchester Guardian, que dieron pié a la elaboración de su obra A Tract on Monetary Reform, aparecida en 1923, referida a los problemas de la inflación, la deflación y las fluctuaciones del tipo de cambio, además de tratar sobre la política monetaria basada en la teoría cuantitativa del dinero, según la versión de Marshall y Pigou.

En 1925, Keynes contrajo matrimonio en Londres con la rusa Lydia Lopokova, bailarina de la compañía Diaghilev, y en la segunda mitad de los años veinte preparó su gran obra A Treatise on Money, publicada en 1930 en dos volúmenes. Antes visitó Madrid en el mes de junio para dar una conferencia en la Residencia de estudiantes a la que más adelante se hará referencia. En el primer volumen del Treatise, The pure theory of Money, Keynes ofrecía soluciones al paro, consistentes en aplicar una política de bajos tipos de interés por parte del Banco Central, con objeto de fomentar la inversión y eliminar el exceso de ahorro, así como reducir las pérdidas empresariales, llevando a un aumento del empleo. En el segundo volumen, The applied theory of Money, describía los principales aspectos de los sistemas crediticios de diferentes países, haciendo hincapié en la importancia de aplicar una política monetaria instrumentada a través del tipo de interés.

Tras las críticas recibidas por esta última obra, Keynes empezó a preparar otro libro que lo catapultó a la fama, publicado en febrero de 1936 con el título 
The General Theory of Employment, Interest and Money, en el que planteaba la novedosa teoría de la demanda efectiva compuesta de tres elementos fundamentales: la teoría del consumo, la eficiencia marginal del capital y la preferencia por la liquidez. Según Keynes, la renta podía quedar determinada en el nivel de equilibrio macroeconómico, aunque podía corresponder a una situación de desempleo, la cual era socialmente indeseable y por ello era preciso corregir. Para lograr el aumento del empleo, y dada la escasa eficacia del manejo del tipo de interés y en general de la política monetaria, Keynes consideraba necesario recurrir a la política fiscal, aunque ello supusiese déficit público y, en consecuencia, romper con uno de los dogmas de la economía clásica, el equilibrio presupuestario.

Además, en la primera mitad de la década de los treinta, Keynes publicó otros dos libros destacados. En 1931 apareció Essays in Persuasion, obra de recopilación de artículos referidos a su actividad como publicista, como fue la conferencia que impartió en 1930 en Madrid "El futuro económico de nuestros nietos”. En 1933, Keynes publicó sus Essays in Biography, que contenía una serie de retratos de distintos personajes: políticos, economistas y científicos.

Su actividad de asesoramiento político y económico, como liberal independiente, fue también muy intensa. En este sentido durante las negociaciones de Bretton Woods en 1944, Keynes representó a la delegación británica, mientras que al frente de la delegación de Estados Unidos estaba White. Ambos representantes mostraban visiones muy diferentes de cómo afrontar los problemas de la posguerra. Aunque finalmente se adoptó el plan propuesto por White, muchas de las ideas del Plan Keynes fueron tenidas en cuenta para la creación del Fondo Monetario Internacional y del Banco Mundial.

Desde principios de los años treinta, Keynes fue recibiendo numerosos reconocimientos entre los que cabe destacar su designación como miembro del Economic Advisory Council en 1930 y su nombramiento como Consejero del Banco de Inglaterra en 1941. Un año después, en 1942, recibió el título de Lord Keynes. Falleció de un ataque al corazón en Tilton (Sussex) el 21 de abril de 1946, con sesenta y dos años. Había llegado a la Economía a través de la Filosofía y se convirtió en un imprescindible personaje de su época. Fue funcionario del Tesoro británico, profesor de Economía, ensayista, periodista, e inversor en bolsa, pero ante todo una personalidad polifacética al servicio de la ciencia económica y de los intereses generales de ámbito internacional.

\section{LAS TRADUCCIONES AL CASTELLANO DE LOS LIBROS DE KEYNES}

Según la búsqueda realizada, se tiene constancia de que al castellano se han traducido, como ya se ha indicado, seis libros de Keynes, publicados tanto en España como en Méjico. 
El trabajo pionero sobre las obras de Keynes traducidas al castellano -el cual nos servirá de guía para esta parte del trabajo-, es el artículo de Juan Velarde "Biblioteca hispana de Marx, Keynes y Schumpeter. Una primera aproximación” (1983). En 1985, bajo el título Ensayos sobre intervención y liberalismo, ediciones Orbis publicaba traducidos por Jordi Pascual, tres ensayos de Keynes contenidos en The Collected Writtings: "The economic consequences of Mr. Churchill” (1925), "The end of laissez-faire” (1926) y "How to pay for the war" (1940). Fueron seleccionados y presentados en esa edición por Antonio Argandoña.

\subsection{The economic consequences of the peace}

Como señalaba el primer biógrafo de Keynes R.F. Harrod ${ }^{2}$, la obra The economic consequences of the peace, fue escrita durante los meses de agosto y septiembre de 1919 en Charleston, y en diciembre de ese año se puso a la venta. En pocos meses se llegaron a vender en el mundo más de 100.000 ejemplares. El libro fue traducido a once idiomas (alemán, holandés, flamenco, danés, sueco, italiano, español, rumano, ruso, japonés y chino). Para $\operatorname{Harrod}^{3}$ era en esos momentos "una de las mejores obras polémicas de la lengua inglesa", y para Skidelsky ${ }^{4}$ se podía considerar entonces el mejor libro de Keynes. A su éxito de ventas se unieron fuertes críticas, de forma que como señalaba Harrod ${ }^{5}$, "Keynes quedó proscrito de los círculos oficiales británicos durante muchos años”. Una de las principales críticas a ese libro de Keynes vino de la mano de Chamberlain, quien le recriminaba a Keynes las tensas referencias que en la obra hacía a su propio país, teniendo en cuenta el destacado papel que Inglaterra había tenido en las negociaciones del Tratado de Versalles ${ }^{6}$.

Esta obra fue la primera de Keynes traducida al castellano, aparecida en Madrid en 1920 por la editorial Calpe con el título Las consecuencias económicas de la paz. Entre 1920 y 2013 se han localizado siete ediciones,

\footnotetext{
2 (1958), p.295.

${ }^{3}$ (1958), p.296.

${ }^{4}$ (2013), p. 345.

${ }^{5}$ (1958), p. 295.

${ }^{6}$ Skidelsky (2013), p. 353. El origen del tema principal de este libro de Keynes se puede encontrar, según Skidelsky, en una observación que le había hecho el ministro de defensa sudafricano,- Jan Christian Smuts, general intelectual licenciado en Cambridge en 1894 y al que Keynes había tratado en París-, sobre la importancia que en el siglo XIX había tenido la prioridad concedida a la reforma económica antes de la política, y que esas circunstancias se estaban repitiendo tras la primera guerra mundial, en el sentido de que las cuestiones políticas y territoriales no se solucionan hasta que no se resuelvan los problemas económicos. Ante esa observación Keynes dijo “que era verdad y que nunca se lo había planteado de esta forma”. Skidelsky (2013), p. 338.
} 
todas ellas con la traducción de Juan Uña Sarthou ${ }^{7}$. Para la traducción al castellano de The economic consequences of the peace, hubo cinco solicitantes. Además de Juan Uña Sarthou, mostraron interés Harold Ballou, Vicente Gay, José Ruiz-Castillo y Juan Guixé. Keynes solicitó referencias sobre Uña a algunos amigos, basándose en la opinión de José Castillejo y Salvador de Madariaga ${ }^{8}$.

Pero antes de ser publicada en castellano, fue objeto de estudio por parte del economista y profesor, el catedrático Luis Olariaga, quien redactó dos artículos para el diario El Sol relacionados con esa obra: el primero, titulado "Un libro sobre la paz. El Tratado de Versalles va a completar la ruina económica de la Europa Continental”, apareció el 28 de enero de 1920, y el segundo, publicado el 4 de febrero de ese mismo año, llevaba por título "Un libro sobre la paz. El Tratado de Versalles deja sin carbón a la industria alemana y a toda Europa Septentrional y Central”. En el primer artículo, Olariaga consideraba que en el fondo, con la política económica contenida en el Tratado de Versalles dictada por M. Clemenceau, -influyente miembro del Consejo de los Cuatro-, no se pretendía más que salvar a Francia, y aniquilar económicamente a Alemania. Estas duras consecuencias según Olariaga, que está de acuerdo con Keynes en sus negativos efectos económicos, conducirían a que la ruina económica de Alemania supondría también la de toda la Europa continental. En el segundo artículo, Olariaga concretaba cuáles eran según el Tratado, las más graves obligaciones económicas impuestas a Alemania, así como sus efectos, que se concretaban en el carbón y en el mineral de hierro, bases de la economía industrial alemana (siderurgia, química y electricidad). En este sentido, señalaba Olariaga que si Alemania cumplía el Tratado, su industria podría desaparecer y no podrían abastecerse de carbón otros países de Europa. De la misma manera, al anexionar a Francia las regiones de Alsacia y Lorena por la aplicación del Tratado, Alemania también reduciría considerablemente su producción de hierro, con los consiguientes efectos de desabastecimiento para otras economías europeas.

Según Velarde ${ }^{9}$, ese mismo año 1920 Olariaga, tras haber comenzado como profesional de la banca en Gran Bretaña en 1905, concretamente en el Crédit Lyonnais, vuelve a ese país fascinado por la aparición del libro de Keynes ${ }^{10}$.

\footnotetext{
${ }^{7}$ Un año después, Juan Uña y Sarthou también tradujo al español la Autobiografía de John Stuart Mill, publicada por Calpe en Madrid en 1921, que lleva un escueto prólogo del propio Juan Uña.

${ }^{8}$ Almenar (2001), p. 794.

${ }^{9}$ Velarde (1989), p. xxvi

${ }^{10}$ Así lo ha señalado el propio Olariaga en una nota manuscrita, fechada en noviembre de 1973, que forma parte del archivo familiar, hoy cedido a la Real Academia de Ciencias Morales y
} 
Asimismo, al asistir Olariaga a la Conferencia de Génova de 1922, en calidad de Consejero técnico de la Comisión española, tuvo allí un encuentro con Keynes. Es muy probable, según Velarde ${ }^{11}$, que ambos tratasen sobre un tema polémico en esos momentos, como era la conveniencia de volver a las paridades de las monedas anteriores a la Primera Guerra Mundial y el retorno al patrón oro.

Una vez publicada en castellano la obra en abril de 1920, otro de los autores que llamó la atención sobre este libro de Keynes fue el escritor y filósofo Eugenio D’Ors, quien le dedicó una glosa a John Maynard Keynes. Fue a raíz de su elección como presidente de la Asociación de la Prensa de Barcelona, en febrero de 1920, cuando comenzó una colaboración semanal titulada "Las obras y los días" en el diario Las noticias, mediante artículos que firmaba como Xenius $^{12}$. Concretamente el 16 de mayo de 1920, publicaba el artículo “Keynes”13, en el que D’Ors señalaba que no debía dejar de leerse Las consecuencias económicas de la paz, una obra de éxito por la lucidez que había mostrado su autor, a quien D’Ors consideraba un precursor.

De esa obra de Keynes, Eugenio D’Ors eligió el siguiente párrafo referido al Tratado de Paz:

"Tejido de sofismas que revistieron de mentira todo el texto y la sustancia del

Tratado, enteramente..." "Los sofistas más insidiosos y los fautores de planes más hipócritas fueron empleados en él" ${ }^{14}$.

Por otra parte, el traductor de esta obra de Keynes Juan Uña, publicó en julio de 1920 una reseña con un preciso mensaje económico en La lectura. Revista de Ciencias y Artes en la que calificaba al libro de Keynes de "humano y noble", así como "aliadófilo"", por la contundencia de su autor tanto al valorar el Tratado de Versalles como a la importancia que le daba a una Europa unida. En este sentido señalaba:

"Keynes afirma en redondo y maneja toda clase de cifras y datos para demostrar que el Tratado es irrealizable. Europa constituye un todo económico: la ruina de una de sus partes implicaría la desorganización y hasta la ruina de las demás"... "Los peligros que amenazan a Europa son la falta de

\footnotetext{
Políticas: "Estudiar las consecuencias económicas de la contienda siguiendo el trazo que iba marcando John Maynard Keynes”. (en Velarde, 2003, p. 85).

${ }^{11}$ Velarde (1989), p. xxix.

${ }^{12}$ González (2010), p. 39.

${ }^{13}$ Este artículo de D’Ors fue recogido en el volumen 1 de su Nuevo Glosario, publicado en Madrid por la editorial Aguilar en 1947, p. 186.

${ }^{14}$ D’ors (1947), p. 186.

${ }^{15}$ Uña (1920), p. 210.
} 
aprovisionamiento, de carbón y de transportes. Europa no podrá vivir sin la generosidad de América" 16 .

A la visión pesimista sobre el futuro de Europa derivada del libro de Keynes $^{17}$, Juan Uña añadía una referencia al informe que el conde BrockdorffRantzau $^{18}$ había redactado el 13 de mayo de 1919 sobre la conferencia de Paz, previamente a la firma del Tratado de Versalles, el cual sostenía que "la ejecución de las condiciones del Tratado sería tanto como condenar a muerte a millones de personas en Alemania” ${ }^{19}$. Para Uña, el pesimismo que mostraba Keynes en ese libro, se podía entender como una "apreciación real de las dificultades humanas y de la lentitud de ese camino”. Por todo ello, Juan Uña terminaba su reseña alabando la figura de Keynes, como un gran revolucionario y un paladín de la verdad ${ }^{20}$.

Por otra parte, en 1921 se publicaron los capítulos I, II y VI de esa obra de Keynes en el volumen IX (28) de la Revista Nacional de Economía, con el título "Las consecuencias económicas del Tratado de Versalles". A pesar de la difusión en España de esta obra, a la editorial Calpe no le pareció muy rentable su venta, ya que en noviembre de 1921 Keynes ofreció la traducción española de A revision of the Treaty (apéndice a Las consecuencias económicas de la paz), pero la editorial Calpe no lo aceptó, con la excusa de que solo se habían vendido en España 700 ejemplares de Las consecuencias económicas de la $\operatorname{paz}^{21}$.

El interés por los efectos económicos de la Gran Guerra ya estaba calando en España, sobre todo a través de la Junta para Ampliación de Estudios, la cual había dotado de financiación una línea de investigación para esa cuestión ${ }^{22}$. Tras un primer intento fallido por parte de Antonio Flores de Lemus, quien a pesar de haber solicitado a la Junta para Ampliación de Estudios una ayuda para estudiar cómo le afectaría a las Haciendas Públicas francesa e inglesa los resultados de la Primera Guerra Mundial, renunció poco después de habérsele concedido la ayuda, y por iniciativa del propio Flores de Lemus, fue encargado el estudio a Francisco Bernis, Catedrático entonces de la Universidad de Salamanca. Fruto de esa ayuda de la Junta, fue la publicación por parte de

${ }^{16}$ Uña (1920), p. 214.

${ }^{17}$ Como señala Sylvia Nasar (2013), p. 287, Las consecuencias económicas de la paz es un libro tremendamente derrotista, motivo por el cual Leonard Woolf apodó a su autor Keynesandra.

${ }^{18}$ Ulrich von Brockdorff-Rantzau, era ministro de Asuntos Exteriores en la República de Weimar.

${ }^{19}$ Uña (1920), p. 216.

${ }^{20}$ Uña (1920), p. 218.

${ }^{21}$ Almenar (2001), p. 797.

${ }^{22}$ En este sentido, Velarde (1989), p.xxvi, apuntaba que los hermanos Ángel y Avelino Gutiérrez, aportaron 1.445 libras esterlinas para impulsar esa línea de investigación. Estas referencias también se pueden leer en la tesis de José Miguel Fernández Pérez (1988), pp. 382-383. 
Bernis del libro Consecuencias económicas de la guerra, aparecido en 1923, con cerca de 400 páginas.

\subsubsection{Cambó, Keynes y Las consecuencias económicas de la paz}

No hay referencias a España en este libro de Keynes, a pesar de haber visitado nuestro país con anterioridad en dos ocasiones: 1907 y 1909. Según Velarde $^{23}$, "Esta ignorancia absoluta de España es para mí muy significativa, en tanto en cuanto, como nos ha recordado Enrique Fuentes Quintana, a partir de su confortable base de Biarritz y en compañía del historiador de la economía Charles Rye Fay, Keynes llega en 1907 a la zona de Torla, en las proximidades del Valle de Ordesa. En junio de 1909 vuelve. A Duncan Grant le llega a escribir: 'Cuando tenga que huir de mi país, será este valle al que me retire a vivir entre truchas, fresas silvestres y pastores españoles’”24.

No obstante, hay una referencia indirecta a España, más concretamente a Cambó, en el capítulo IV de Las consecuencias económicas de la paz, relativa al Tratado de Versalles. En él, Keynes exponía las principales medidas económicas contenidas en ese Tratado, indicando cómo aspiraba éste a destruir los principales pilares del sistema económico alemán: su comercio marítimo, la explotación de su carbón y de su hierro, así como las industrias basadas en ellos.

Al hacer referencia al artículo 235 del Tratado de Versalles, según el cual se autorizaba a la Comisión de reparaciones desde el 1 de mayo de 1921 a exigir a Alemania el pago de 1.000 millones de libras en la forma que quisiera fijar este país, -podría ser en oro, mercancías, barcos, valores o de otra manera-, Keynes interpretaba que este artículo del Tratado habilitaba a la Comisión de reparaciones a fijar como objetivo "cualquier negocio especial, empresa o propiedad, bien dentro o fuera de Alemania, y pedir su entrega”25. En este sentido, Keynes ponía como ejemplo y daba por hecho que sería intervenida "la magnífica y poderosa empresa alemana de Sudamérica conocida por la Deutsche Ueberseeische Elecktrizitatsgesellschaft (DUEG) y disponer de ella en servicio de los intereses aliados”26. Para Keynes, más que castigar a Alemania, lo que había que hacer era reconstruir Europa. Keynes acertó, por tanto, en su visión futura del Tratado. Humillar a Alemania al exigirle el pago de unas elevadas reparaciones por la Gran Guerra, implicaba en el fondo hacer de Alemania una gran potencia económica mundial ${ }^{27}$.

23 (1989), p. 106.

${ }^{24}$ La referencia a Fuentes Quintana corresponde a su artículo sobre Keynes en España de 1983.

${ }^{25}$ Se cita por la versión castellana de Keynes (2012), p. 78.

${ }^{26}$ Keynes (2012), p. 78.

${ }^{27}$ Como es sabido, Alemania terminó de pagar esas reparaciones de la Primera Guerra Mundial en octubre de 2010 . 
Las casualidades de la vida hicieron que, curiosamente, al mencionar Keynes a la empresa alemana DUEG en Las consecuencias económicas de la paz, como se deduce de la cita recogida en el párrafo anterior, ello condujera a que Cambó acumulase una gran fortuna al hacerse cargo de la presidencia de la Compañía Hispano-Americana de Electricidad (CHADE). Pero, ¿cómo llegó Francesc Cambó a presidir esta Compañía? Según Jesús Pabón ${ }^{28}$, biógrafo de Cambó, el proceso se inició a raíz de las posibles consecuencias negativas sobre la empresa DUEG, -mencionada por Keynes en su obra-, y por la decisión tomada por el representante principal de esa empresa Walter Rathenau, de constituir a toda prisa una nueva sociedad hispanoamericana con los activos de la DUEG y que fuese domiciliada en alguno de los países neutrales durante la Gran Guerra, como era el caso de España ${ }^{29}$. La DUEG consultó con sus empresas participantes, entre ellas la sociedad belga SOFINA, con domicilio social en Bruselas, que era uno de los principales accionistas de la DUEG. La opinión del principal representante de esta sociedad belga, Heineman, fue la de crear una compañía que se hiciera cargo de los negocios de la DUEG en América, opinión que se tuvo muy en cuenta y fue decisiva para la creación de la CHADE. La relación con Cambó procedía de su amistad, especialmente con Heineman, y también con Rathenau. El primero, consultaba a Cambó como abogado sobre los asuntos que tenía en España, país que conocía desde 1905; en cuanto a Rathenau, que también conocía a Cambó, valoró positivamente la destacada labor realizada por este político, lo que dio la suficiente confianza a la empresa DUEG para responsabilizar a Cambó como presidente de la nueva Sociedad. En las gestiones previas para la creación de la CHADE, Cambó contó con la ayuda de Juan Ventosa y Miquel Vidal y Guardiola, así como de algunos bancos como Urquijo, Central, Vizcaya y Barcelona. Finalmente la CHADE se fundó en Madrid el 22 de junio de 1920 con un capital de 120 millones de pesetas, del cual la mitad correspondía a acciones entregadas en canje de las antiguas acciones de la DUEG alemana, y la otra mitad fueron acciones suscritas por Cambó y algunos bancos como el Urquijo. A partir de ese momento Cambó, como presidente de la CHADE, se convirtió en un hombre rico y ello le permitió desarrollar diversas actividades culturales y filantrópicas, entre las que cabe destacar la Fundaciò Bernat Metge, dando lugar a la creación de un magnífico archivo y una biblioteca ${ }^{30}$.

${ }^{28}$ (1969), vol.II, pp. 217-226.

${ }^{29}$ Hay que tener en cuenta que la DUEG, en enero de 1898, construyó una fábrica de electricidad en Buenos Aires, y en los diez primeros años del siglo XX había extendido sus actividades en Argentina, Chile y Uruguay (Pabón, 1969, p. 221).

${ }^{30}$ Otro de los resultados fue la apertura por Cambó de un conocido despacho en la Vía Layetana de Barcelona, en el que años después entraría a trabajar Lucas Beltrán como secretario particular de Cambó (Huerta de Soto, 1996, p. xv). 


\subsection{A Tract on Monetary Reform}

Tras la traducción al castellano de The economic consequences of the peace en 1920, la obra de Keynes de 1923 A Tract on Monetary Reform, no fue traducida y publicada en castellano hasta 1992, es decir, 69 años después de su versión original inglesa, con el título Breve Tratado sobre la reforma monetaria, editada en Méjico por el Fondo de Cultura Económica. A esa primera traducción castellana le ha seguido una última más reciente publicada en 2009 por la Fundación ICO y la editorial Síntesis, que contiene en la misma edición un conjunto de escritos de Keynes publicados entre 1910 y 1944. Su título es Breve tratado sobre la reforma monetaria. Escritos (1910-1944) y lleva una introducción de Antonio Torrero. Respecto a las referencias a España en esta obra, solo hay una mención concreta cuando Keynes trata sobre los efectos positivos para un país de la restauración de la paridad oro de su moneda al nivel anterior a la primera guerra mundial, al dar prestigio financiero a la nación y una mayor confianza en su futuro. En este sentido, Keynes se refiere a España aunque con cierta duda, así como a Gran Bretaña, Holanda, Suecia y Suiza.

\subsection{A Treatise on Money}

La primera y única traducción autorizada en lengua española de esta obra de Keynes de 1930 es muy reciente. Fue publicada en 2010 por la Fundación ICO, con el aval de la Royal Economic Society y la Editorial Macmillan. Como valor añadido, cuenta con una extensa introducción del Profesor Torrero. Asimismo, esta traducción ve la luz en unas circunstancias financieras y económicas parecidas a las que hicieron que $A$ Treatise on Money fuese publicado en Londres. Entonces fue la Gran Depresión y su traducción en lengua española ha sido publicada en 2010, en plena crisis financiera y económica.

Entre los aspectos fundamentales de esta obra, Torrero ${ }^{31}$ ha señalado que se trata de un libro de teoría monetaria, que Keynes había estado preparando de manera intermitente durante los seis años anteriores a su publicación, etapa fundamental en la evolución de su pensamiento. La experiencia acumulada durante los años veinte, le sirvió a Keynes para dar una nueva explicación teórica de cómo se desarrollan las fases del ciclo económico en base a la evolución y los beneficios de las empresas. Con el estallido de la Gran Depresión, poco antes de su publicación, Keynes introdujo algunas modificaciones para adaptarlo a las nuevas circunstancias aunque, como señala Torrero ${ }^{32}$, "se percibe una cierta falta de unidad en el libro y el propio Keynes confesó su incomodidad...; el contenido del Tratado no respondía con exactitud al pensamiento de Keynes cuando el libro se publicó". A ello se unió otra circunstancia, como fue su nombramiento como

${ }^{31}$ (2010), pp. xv-xix.

32 (2010), pp. xv-xvi. 
miembro del Comité MacMillan ${ }^{33}$, cuando el libro se encontraba en imprenta. Con la experiencia adquirida en ese Comité, Keynes modificó y pulió sus puntos de vista, entre ellos la importancia que empezó a conceder al factor empresarial como elemento determinante del progreso económico ${ }^{34}$. Este aspecto se considera fundamental en el presente trabajo, por estar relacionado con las referencias que Keynes hizo a España en su Tratado.

\subsubsection{El Tratado sobre el dinero y España}

En el Tratado sobre el dinero de Keynes, se menciona a España en los capítulos 30, 33, 35 y 36 de la obra. El profesor Fuentes Quintana ${ }^{35}$ resume el contenido de esta obra de Keynes y sus referencias a España a través del proceso histórico de la llegada a Europa del tesoro español de Indias. En este sentido señalaba que:

"con este ejemplo histórico de la difusión de los efectos del Tesoro español, Keynes intentaba ilustrar su teoría de que la acumulación de capital depende de las expectativas de beneficios de los empresarios y de las posibilidades de financiación de los proyectos de inversión por el sistema monetario y bancario" 36.

En el capítulo 30, titulado Ejemplos históricos, Keynes se refería al objetivo principal de su obra, centrado en explicar el ciclo económico ligándolo a los beneficios y pérdidas de las empresas. Para Keynes ${ }^{37}$, el sacrificio en el consumo por parte de los individuos no es una condición suficiente para elevar la renta y la riqueza, ya que ello podía provocar efectos desfavorables sobre los beneficios. Para ampliar este argumento central de su obra, Keynes tomaba ejemplos de algunos episodios de la historia de los precios, así como del impulso que recibieron algunas civilizaciones a través de la riqueza de sus metales monetarios, como fueron los casos de la civilización árabe, Sumeria, Egipto, Atenas y Roma. Respecto a esta última, Keynes hacía una referencia a España al señalar:

"Los grandes suministros de metales preciosos procedentes de las minas españolas propiedad de Roma también fueron, por supuesto, un elemento vital

${ }^{33}$ Este Comité fue constituido el 5 de noviembre de 1929. Entre sus miembros se encontraban además de Keynes, Pigou, Robbins y Robertson. Uno de sus principales objetivos era tratar de reactivar la economía inglesa mediante una expansión monetaria sin causar tensiones inflacionistas. El informe fue publicado en junio de 1931.

${ }^{34}$ En este sentido, Torrero recoge una cita de Clarke (1988), p.p.107-108, según la cual en un añadido de última hora, Keynes modificó en el Tratado sobre el dinero el protagonismo del progreso económico, haciéndolo depender mucho más de la pujanza empresarial.

35 (1983), pp. 264-265.

${ }^{36}$ Fuentes Quintana (1983), p. 264.

${ }^{37}$ Se cita por la version castellana de Keynes (2010), p. 469. 
en el sistema económico romano, después de que se arrebatara Sierra Morena a Aníbal" 38 .

Por otra parte, Keynes se refería al éxito de la expedición de W. Phipps frente a las costas de La Española, que logró recuperar un barco español hundido, operación que permitió rescatar entre 250.000 y 300.000 libras. Llegada a Londres esta expedición en 1.688, los efectos económicos de este importante acontecimiento para Inglaterra, fueron extraordinarios según Keynes, ya que influyó en la subida de la bolsa de valores inglesa durante los años 1692-1695, y por otra parte, fue determinante en la fundación del Banco de Inglaterra.

A continuación, en el mismo capítulo 30, apartado 1, titulado El tesoro español, Keynes profundizaba en el episodio de la inflación vivido en Europa durante los siglos XVI y XVII, tras la llegada de metales preciosos a España, basándose para ello en diversos artículos publicados por Earl Hamilton a finales de los años veinte ${ }^{39}$. Así, Keynes se refería a la llegada a España en 1519 de los primeros tesoros aztecas procedentes de México, el botín inca de Pizarro llegado en 1534 desde Perú y, una década después, la nueva producción de las minas de Potosí. El fuerte ascenso de los precios en España, como recoge Keynes en su Tratado sobre el dinero, comienza en 1519 con la subida de los precios andaluces, la cual se extendió durante las 8 décadas siguientes. Para llegar a esta conclusión, Keynes se basó en la información recogida por Hamilton de los contables de la Casa de Contratación de Sevilla, creada en 1503, y como resumía el propio Keynes ${ }^{40}$ :

"Así pues, digamos que los años comprendidos entre 1550 y 1600 fueron el periodo de cambios revolucionarios de los precios y que hacia 1630 había concluido esta fase de la historia monetaria”.

Pero no terminan aquí las referencias a España en este capítulo. Como la intención de Keynes era corroborar su hipótesis de que la riqueza de las naciones se eleva cuando los precios superan ampliamente a los costes, tenía que recopilar datos sobre esta última magnitud, y para ello el único indicador disponible era la evolución de los salarios. Para el caso de España, la explicación que daba Keynes $^{41}$ se basaba en el índice de salarios españoles recogidos por Hamilton en el artículo de Economica de 1929. En síntesis, las condiciones para la acumulación de la riqueza en España, según la tesis de Keynes, solo se dieron entre 1520 y 1560; y a partir de 1588 los salarios españoles no solo fueron superiores a los precios, -lo que motivó una deflación de beneficios,- sino que crecieron situándose por encima de los del resto de Europa. A ello se unió en opinión de Keynes, una circunstancia que agravó la situación, como fue la

\footnotetext{
${ }^{38}$ Keynes (2010), p. 471.

${ }^{39}$ Hamilton (1928, 1929a, 1929b, 1929c)

${ }^{40}$ Keynes (2010), p. 472.

${ }^{41}$ Keynes (2010), p. 474.
} 
pérdida de población debido a varios motivos: ingreso en el ejército, emigración a América, elevado número de personas célibes, expulsión de los moriscos, así como la emigración hacia las ciudades de los campesinos, bien para dirigirse a ultramar o para aumentar su poder adquisitivo mediante la realización de servicios personales.

Sin embargo, en el caso de Inglaterra y Francia, la evolución de los salarios fue muy diferente a la de España, consiguiendo estos países elevar su riqueza durante el siglo XVII. De nuevo Keynes vuelve a referirse a España al reconocer que en el aumento de la riqueza de Inglaterra, la piratería había jugado un decisivo papel, al proporcionar una gran parte de las importaciones de metales preciosos. En este sentido Keynes señalaba:

"De hecho, el botín traído por Drake en el Golden Hind puede considerarse con justicia la fuente y el origen de la inversión exterior británica. La reina Isabel devolvió con los ingresos toda su deuda exterior e invirtió una parte del resto (alrededor de 42.000 libras) en la Compañía de Levante; con los beneficios de esta compañía se creó en gran medida la Compañía de las Indias Orientales”"

Al final del capítulo 30 del Tratado sobre el dinero, Keynes llegaba a una serie de conclusiones que resumimos a continuación:

1) Está más convencido de que es mejor evitar las deflaciones de beneficios que desear las inflaciones de beneficios.

2) Lo anterior se relaciona con los negativos efectos de la inflación de beneficios sobre la distribución de la riqueza en una economía, haciéndola más desigual, lo que a juicio de Keynes se contrarrestaría "mediante impuestos directos sobre los ricos, como los que hay actualmente en Inglaterra" ${ }^{43}$.

3) La tasa de crecimiento del capital debe convertirse en un asunto de Estado con una visión a largo plazo. De esta manera se evitaría el dilema entre ahorro y beneficios como medios para lograr el crecimiento de la riqueza de un país.

4) El objetivo principal de toda política económica debería ser doble: evitar la deflación y aspirar a la estabilidad del poder de compra.

En cuanto al capítulo 33 del Tratado sobre el dinero, titulado Métodos de gestión nacional, en el apartado I, dedicado a los Métodos existentes para regular las emisiones de billetes, Keynes vuelve a referirse al caso español al tratar del sistema porcentual, calificándolo como el "más moderno hoy en día” ${ }^{44}$. Para ello, en una tabla se mostraban los porcentajes mínimos de reservas de oro sobre la emisión de billetes, establecidos en España y en otros once

\footnotetext{
${ }^{42}$ Keynes (2010), p. 475.

${ }^{43}$ Keynes (2010), p. 479.

${ }^{44}$ Keynes (2010), p. 560.
} 
países (entre ellos Alemania, Estados Unidos y Francia). En conjunto para esos países, los porcentajes oscilaban entre el 30 y el 40\%. Concretamente España tenía asignado en esa tabla un $37 \%$, y este porcentaje iba acompañado de la nota al pie siguiente: "el sistema español es complicado, ya que el porcentaje exigido aumenta a 47 cuando la emisión de billetes supera una determinada cifra” ${ }^{\text {". }}$.

Otro de los análisis incorporado por Keynes en este mismo apartado 1 del capítulo 33 era averiguar el conjunto de reservas de oro que tenían en ese momento los principales bancos centrales del mundo, distinguiendo entre las "reservas mínimas legales" (inmovilizadas a efectos prácticos) y el exceso de reservas (para hacer frente a las emergencias). A tal efecto recogía los datos de nueve países, entre ellos España, el cual aparecía con unas reservas legales de 55 millones de libras y un exceso de reservas de 32 millones de libras ${ }^{46}$. Keynes expresaba una cierta preocupación al constatar el elevado porcentaje de las reservas mundiales de oro que se encontraban inmovilizadas en los bancos centrales (casi dos tercios) y el reducido exceso de reservas de oro, con el consiguiente riesgo de que ante periodos de escasez de oro se endureciesen las condiciones del crédito y cayese el nivel de precios ${ }^{47}$.

Respecto al capítulo 35 del Tratado sobre el dinero, titulado Métodos de gestión internacional. II. El patrón oro, Keynes se refiere a España en dos ocasiones de forma puntual: una para señalar que se había exagerado la cantidad de oro que había entrado en España procedente de Sudamérica, según las investigaciones de Hamilton; y otra para hacer referencia a la cantidad de oro existente en los bancos centrales de diversos países, entre ellos España.

Finalmente, en el capítulo 36 del Tratado sobre el dinero, titulado Métodos de gestión internacional, Keynes se refería en el apartado III, titulado El problema de la autonomía nacional, a las diferencias existentes en esos momentos en algunas economías europeas, entre ellas España, entre la política monetaria aplicada y el distinto conocimiento sobre temas monetarios del público en general. En este sentido señalaba:

"La actitud actual del Banco de Inglaterra o del Reichsbank hacia el oro es fundamentalmente diferente de la actitud del Banco de Francia o del Banco de España y que los cambios para los que los primeros pueden estar maduros en los próximos cinco o diez años podrían ser aún demasiado nuevos para los segundos $" 48$.

\footnotetext{
${ }^{45}$ Keynes (2010), p. 561.

${ }^{46}$ Para hacer esos cálculos, Keynes supuso un cambio de 30 pesetas por libra hacia 1928.

${ }^{47}$ Keynes (2010), pp. 563-564.

${ }^{48}$ Keynes (2010), p. 616.
} 


\subsubsection{Luis Olariaga y el Tratado sobre el dinero de Keynes}

Otra relación entre el Tratado sobre el dinero de Keynes y España, es la procedente de la recepción española de esa obra realizada por el Profesor Olariaga que, según Velarde ${ }^{49}$, fue el primero en llamar la atención a los españoles sobre las ideas de Keynes. Concretamente sobre esta obra de Keynes se ocupó Olariaga en su destacado libro La política monetaria en España, publicado en 1933.

Según Juan Velarde ${ }^{50}$, se pueden resumir en cuatro apartados las principales referencias de Olariaga al Tratado sobre el dinero de Keynes:

1) Sobre la capacidad de compra del dinero, Olariaga señalaba el rechazo de Keynes a la forma en que se medía en esos momentos. Keynes consideraba que debía utilizarse un número índice que incluyera directa o indirectamente, todo cuanto entra en el consumo final.

2) En relación al tipo de interés, Olariaga recogía la influencia que había tenido sobre Keynes el planteamiento realizado por Knut Wicksell en su obra Geldzins und Güterpreise, publicada en Jena en $1898^{51}$. Para Keynes, si bien el tipo de interés era eficaz para corregir los desequilibrios en el nivel de precios si éstos se originan con la inflación de beneficios, no servía para lograr una deflación de ingresos (salarios) cuando éstos eran los causantes de las presiones inflacionistas. Ello venía motivado principalmente por la resistencia que los sindicatos estaban ya mostrando a una bajada de salarios, en la segunda mitad de la década de los veinte ${ }^{52}$.

3) En cuanto a algunas de las peculiaridades del sistema monetario inglés, Olariaga destacaba la llamada de atención de Keynes sobre la escasa circulación de moneda en Inglaterra durante el periodo 1926-1928 (solo un $10 \%$ del total del dinero circulante), frente al mayor uso del cheque y las transferencias entre cuentas bancarias ${ }^{53}$.

4) Respecto a la reserva bancaria, este tema era objeto de atención por parte de Olariaga debido a la polémica española existente a finales de los años 20, por la depreciación de la peseta frente a la libra. Olariaga recogía la postura de Keynes sobre la finalidad de una reserva de oro, que a su

\footnotetext{
${ }^{49}$ (1988), p. 119.

50 (1988), pp. 121-124.

${ }^{51}$ Cabe recordar aquí que Wicksell distinguía por una parte, entre el tipo de interés natural, que es el que equilibra el sistema económico y, por otra parte, el tipo de interés de mercado o monetario, que es el que cobran los Bancos en los préstamos y créditos que conceden. La discrepancia entre ambos tipos, desencadenará un proceso bien depresivo o inflacionista en la economía.

${ }^{52}$ Torrero (1998), p. 644.

${ }^{53}$ Velarde (1988), p. 121
} 
entender es doble: suministrar recursos líquidos en caso de excepcional necesidad, e inspirar confianza por razones puramente psicológicas ${ }^{54}$.

Puede decirse en resumen que Olariaga, a pesar de sus críticas a Keynes, está de acuerdo con ciertas tesis defendidas en su Treatise on Money, de tal manera que según Velarde ${ }^{55}$ "un lejano eco del Treatise lo tiene la distribución en materias de los dos volúmenes de El dinero de Olariaga”. Para Torrero ${ }^{56}$, Olariaga recoge en su libro La política monetaria en España, varios aspectos fundamentales en los que se muestra crítico con la obra de Keynes: uno se refiere a la explicación que daba Keynes sobre la disminución de las oportunidades de inversión ${ }^{57}$, cuando señalaba al respecto el propio Keynes que "el mundo ha de saturarse de capital fijo y tendrá que dedicarse a atesorar"58, a lo que Olariaga argumentaba que "incluso si se llegara a situación tal que el término medio de las nuevas inversiones de capital no rentase nada, algunas de dichas inversiones rentarían algo y muchas esperarían a rentar" ${ }^{\text {"59; }}$ otro es el relajamiento de la disciplina presupuestaria y de los controles sobre la expansión monetaria y crediticia $^{60}$; también la desconexión del tipo de interés de las variables reales ${ }^{61}$, así como el escaso papel que para Keynes tenían las innovaciones y el progreso técnico, una cuestión ya señalada por los editores de la Teoría General en su segunda edición ${ }^{62}$.

No obstante, para Torrero es significativo que Olariaga coincidiese con Keynes en cuanto a la inquietud mostrada por las implicaciones que para la política económica tenía "la rigidez de las economías modernas con precios fijos o administrados en sectores y factores que dificultan el ajuste, aspecto en el cual Keynes puso especial énfasis” ${ }^{\text {. }}$.

\subsection{Essays in Persuasion}

Este libro de Keynes, publicado inicialmente en 1931, recoge un conjunto de artículos suyos aparecidos en diversos diarios, correspondientes a distintas actividades de asesoramiento político y económico, así como capítulos de libros

\footnotetext{
${ }^{54}$ Estas ideas de Keynes están tomadas del volumen II del Treatise on Money, 1930, pp. 263-265, según indica Velarde (1988), pp. 123-124.

${ }^{55}$ Velarde (1988), p. 121.

${ }^{56}$ (1998), p. 173.

${ }^{57}$ Olariaga (1947), pp. 229-230.

${ }^{58}$ Olariaga (1947), p. 230.

${ }^{59}$ Olariaga (1947), p. 230.

${ }^{60}$ Olariaga (1953), pp. 121-122.

${ }^{61}$ Olariaga (1947), p. 231.

${ }^{62}$ Olariaga (1947), pp. 229-230.

${ }^{63}$ Torrero (1998), pp. 173, pp. 756-757.
} 
pertenecientes a The economic consequences of the peace (1919), A revisión of the Treaty (1922), A Tract on Monetary Reform (1923) y A Treatise on Money (1930).

La intención de Keynes con esta recopilación de trabajos quedó bien clara en el primer párrafo de su prefacio:

"He aquí coleccionados algo así como los graznidos de un cuervo o los cantos de una rana a lo largo de doce años: graznidos o cantos de una Casandra que nunca pudo influir en el curso de los acontecimientos a lo largo del tiempo. El volumen podría haberse titulado "Ensayos de profecía y persuasión", porque la profecía, por desgracia, ha tenido más éxito que la persuasión. Pero fue con un espíritu de persuasión que se escribieron la mayoría de estos ensayos, en un intento de influir sobre la opinión pública" ${ }^{\prime 4}$.

Skidelsky ${ }^{65}$ ha interpretado que con ese párrafo Keynes daba publicidad de sí mismo.

En 1972, la Royal Economic Society incorporó a la edición de 1931 otros dos escritos de Keynes: el primero, titulado Los medios para la prosperidad, corresponde a una recopilación de artículos publicados en 1933 en The Times y en New Statesman and Nation en 1933; y el segundo, titulado Cómo pagar la guerra, fue un libro publicado en 1940 compuesto por dos largos artículos escritos por Keynes para The Times, aparecidos en noviembre de 1939.

Respecto a las traducciones al castellano de esta obra con el título Ensayos de Persuasión, se han encontrado cuatro. Las dos primeras fueron publicadas en Barcelona, una en 1988 por la editorial Crítica con traducción de Jordi Pascual, y la otra en 1997 por la editorial Folio. Las dos últimas se han editado en Madrid, una en 2009 por la Fundación ICO y la editorial Síntesis -por la que se cita en este trabajo- y la más reciente en 2010, por ediciones El País.

Las referencias a España en esta obra de Keynes se encuentran en el capítulo 25, titulado "Las posibilidades económicas de nuestros nietos", título de la conferencia pronunciada en Madrid el 10 de junio de 1930. Esa conferencia no era un tema nuevo para Keynes, sino una adaptación y actualización de otras tres intervenciones suyas: la primera fue en marzo de 1928 en la Sociedad de Ensayos del Winchester College; la segunda tuvo lugar en 1930 en el Club de Economía Política de Cambridge; y ya en vísperas de su llegada a Madrid, la tercera fue ante los Apóstoles el 31 de mayo de 1930, que escucharon a Keynes acerca de su particular visión del futuro. En octubre de ese mismo año, los días 11 y 18, el texto de la conferencia de Madrid fue publicado en Nation and Athenaeum y finalmente, una vez perfilada su redacción definitiva, fue incluida en Essays in Persuasion.

\footnotetext{
${ }^{64}$ Se cita por la versión castellana de Keynes (2009), p. 17.

65 (2013), p. 654.
} 
En su conferencia, Keynes hizo un preciso diagnóstico sobre la situación económica mundial en esos momentos tras el crack de 1929, contraponiendo su punto de vista a la opinión generalizada según la cual, había finalizado para siempre la época de progreso económico característico del siglo XIX. Para Keynes los motivos de ello eran básicamente tres: la mayor velocidad en el aumento de la eficiencia técnica que en la que se desarrolla para absorber los puestos de trabajo; la rapidez en el aumento del nivel de vida; y las trabas a la reducción de los tipos de interés por parte de los sistemas monetario y bancario.

A pesar de los inconvenientes que se deducían de esa situación, Keynes se mostraba optimista y en su conferencia hacía una previsión halagüeña sobre el futuro económico a largo plazo. Para Keynes, el progreso iba unido a dos factores esenciales: las mejoras técnicas y la acumulación de capital. En relación a este último factor se halla la referencia a España ${ }^{66}$, al apoyarse Keynes en el ejemplo del inicio de la era moderna con el descubrimiento del nuevo mundo y la llegada de oro y plata a España, por la importancia que estos hechos tuvieron para la acumulación de capital que tuvo lugar a partir del siglo XVI. Como ya se ha señalado, este ejemplo sobre España fue utilizado por Keynes en su Treatise on Money obra que, por otra parte, estaba terminando cuando vino a Madrid. Según explicaba en su conferencia, esa acumulación de capital tuvo para Keynes un doble origen: de un lado el aumento de los precios $\mathrm{y}$, de otro, los beneficios derivados de esas remesas de metales preciosos que, como explicaba para el caso de Inglaterra, había impulsado la inversión británica en el extranjero cuando, en 1580, Drake robó a España uno de sus tesoros, que fue trasladado a Inglaterra en el Golden Hind.

En la versión definitiva de la conferencia de Keynes en Madrid y según nos recordó Lucas Beltrán ${ }^{67}$, se suprimieron algunos párrafos, como uno que estaba dirigido a España con cariñosas palabras:

"Me figuro que en este siglo de oro -si a tal llega- es muy posible que España vuelva a ocupar el rango que le corresponde como príncipe de las artes, de la civilización y de la vida. Me parece que vuestra civilización y vuestras tradiciones resistirán al trastorno, originado por este nuevo estado de cosas, mejor que las del Norte de Europa y Estados Unidos de América. Porque España -dicho sea en alabanza suya- se ha negado a dedicar todo su esfuerzo a la lucha para lograr, en carrera acelerada, la plétora económica. Los que con todo afán persiguen el dinero, es posible que nos traigan a todos la abundancia económica; pero serán las naciones que hayan sabido conservar su vida, cultivando con perfección cada vez mayor el arte de vivir, las que podrán disfrutar de la abundancia económica cuando ésta llegue”.

\footnotetext{
${ }^{66}$ Keynes (2009), p. 329.

${ }^{67}$ (1989), p. 97.
} 


\subsection{Essays in Biography}

Este libro de Keynes publicado en 1933, es una recopilación de retratos impresionistas de distintos personajes. En él se recogen semblanzas de políticos, como Lloyd George y Churchill, vidas de economistas, como Malthus, Jevons, Marshall y Edgeworth, los científicos Newton y Einstein, y una especie de autobiografía de la etapa juvenil de Keynes.

La única edición en castellano con traducción de Octavi Pellissa, corresponde a la publicada en Barcelona en 1992 por la editorial Crítica. Como se recoge en una nota al pie de esta edición castellana, se trata de una selección de la publicación inglesa de 1972 de Essays in Biography, la cual corresponde al volumen X de The Collected Writtings of John Maynard Keynes. En la edición castellana se omitieron escritos de Keynes sobre algunos amigos y colegas académicos, como el dedicado a Frank Ramsey. No se han encontrado referencias a España en esta obra de Keynes.

\subsection{The General Theory of Employment, Interest and Money}

Por orden cronológico, este libro fue el segundo de Keynes en ser traducido al castellano, con el título Teoría General de la ocupación, el interés y el dinero. La primera edición se publicó en 1943 en México por el Fondo de Cultura Económica, siete años después de la primera versión inglesa, con traducción de Eduardo Hornedo. Pasaron por tanto más de dos décadas entre la aparición de Las consecuencias económicas de la paz y la Teoría General de Keynes, lo que da idea de la existencia en España de un amplio paréntesis, caracterizado por graves carencias en cuanto a la influencia de Keynes y de otros autores como Pigou y Robertson, cuyos trabajos tampoco fueron traducidos en esa etapa. Entre las excepciones cabe señalar la traducción por Evenor Hazera de la importante obra de Marshall Principles of Economics, con el título de Principios de Economía, publicada en Barcelona por Consultor Bibliográfico en 1931. En este sentido, como ha señalado Almenar ${ }^{68}$ : "El reducido número de libros de Keynes traducidos al castellano (hasta la General Theory) aporta un primer rasgo que caracteriza, negativamente, el nivel inmediato de su difusión directa en España”. Tras la primera traducción al castellano en 1943, se han contabilizado hasta 23 ediciones -o reimpresiones la mayoría-, siendo la última la publicada en 2014 por Fondo de Cultura Económica, según aparece en el anexo al final de este trabajo.

Las referencias a España en la Teoría General se encuentran en el capítulo 23, dedicado al Mercantilismo, la usura, el dinero y el subconsumo. Para entender mejor las relaciones que Keynes planteaba en este capítulo entre las ideas mercantilistas y la usura, es necesario mencionar la postura de Keynes

68 (2001), p.790. 
sobre los tipos de interés ${ }^{69}$. En general, su defensa de unos tipos de interés bajos, era para favorecer la inversión, además de permitir una mejora en la distribución de la renta. Asimismo se puede hablar de una evolución de las ideas de Keynes en torno a su visión sobre los tipos de interés. En este sentido, a principios de la década de los años veinte, los tipos de interés significaban para Keynes una variable reguladora del ciclo económico y aún no consideraba que hubiese que adoptar una política permanente de tipos de interés bajos. Pero conforme la deflación y el desempleo fueron aumentando, va consolidando la idea de que hay que mantener de forma permanente bajos tipos de interés, salvo excepciones relacionadas con la lucha contra la inflación y la especulación. Un ejemplo de ello fue la preocupación de Keynes por la escasa vitalidad económica de Gran Bretaña en los años veinte, una situación que achacaba a los efectos deflacionistas derivados de la vuelta al patrón oro de la libra en 1925, ya que la sobrevaloración que ello impuso a la moneda inglesa, obligaba a ser compensada con la reducción de los costes de producción. Esta situación obligó a Gran Bretaña a abandonar el sistema de patrón oro a principios de los años 30. Sobre el riesgo de incurrir en inflación, hubo entre Robertson y Keynes un cierto enfrentamiento, ya que para Robertson las tensiones inflacionistas podrían aparecer si, de manera permanente, se fijase el tipo de interés por debajo de la tasa de beneficios.

Por otra parte, según Skidelsky ${ }^{70}$, Keynes tenía odio a los rentistas ya que como inversor, mostraba su preferencia por las acciones respecto a la deuda pública, ya que como es sabido, fue un reconocido "jugador de bolsa”. Al titular de bonos, Keynes lo llamaba "usurero medieval” o Shylok, que era un personaje del mercader de Venecia de Shakespeare que pide a Antonio el mercader, una libra de su propia carne si éste no cumple con los términos de un préstamo que le ha concedido.

Retomando las ideas del capítulo 23 de la Teoría General, Keynes relaciona la doctrina mercantilista con los tipos de interés bajos y éstos con la usura.

A la primera relación llega Keynes con el argumento sobre el fomento de la riqueza según la doctrina mercantilista, uno de cuyos principios fundamentales era lograr una balanza comercial favorable. En este sentido señalaba que, en condiciones de laissez-faire, el proceso de aumento de la riqueza de un país podría interrumpirse por la insuficiencia de estímulos sobre nuevas inversiones. Esos estímulos, explicaba Keynes, pueden aplicarse en la inversión total, formada de la inversión doméstica o inversión interior y la inversión exterior (incluyendo en ésta la acumulación de metales preciosos). Para Keynes, la inversión interior venía determinada a largo plazo por el tipo de interés

\footnotetext{
${ }^{69}$ Para profundizar en esta cuestión véase Torrero (1998), pp. 856-884 y (2013), pp. 89-98.

${ }^{70}$ (2013), p. 1062.
} 
nacional, mientras que en la inversión exterior influía el saldo favorable de la balanza comercial. De esta forma, en épocas en las que las autoridades no tenían control directo sobre los tipos de interés nacionales o sobre los estímulos a la inversión nacional, las medidas para elevar ese saldo favorable eran el único medio directo de que disponían para aumentar la inversión extranjera y, a la vez, el efecto de una balanza comercial favorable sobre la entrada de metales preciosos era su único medio indirecto de reducir los tipos de interés nacionales, y estimular así la inversión doméstica ${ }^{71}$.

Pero Keynes también añadía cuáles eran las dos limitaciones de esa exitosa política. La primera se producía cuando los tipos de interés nacionales son tan bajos, que al hacer aumentar tanto la inversión como el empleo, pueden llevar a una desmesurada elevación de los salarios. En ese momento, el aumento de los costes interiores comenzará a tener efectos negativos sobre el saldo de la balanza comercial. La segunda limitación ponía en relación, según Keynes, a los tipos de interés nacionales con los extranjeros, de manera que si los primeros se reducen considerablemente respecto a los segundos provocando un desproporcionado aumento de los préstamos al extranjero, ello podía ocasionar una salida de metales preciosos que neutralizaría los esfuerzos realizados previamente para lograr una balanza comercial favorable ${ }^{72}$.

Para ilustrar los argumentos anteriores, Keynes se apoyaba en los casos de varios países, -uno de ellos España- y también Gran Bretaña y la India. El caso de España servía para explicar la primera limitación antes señalada. En este sentido Keynes indicaba:

"La historia económica de España de fines del siglo XV y durante el XVI da el ejemplo de un país cuyo comercio exterior fue destruido por el efecto de una excesiva abundancia de metales preciosos sobre la unidad de salarios" ${ }^{73}$.

El ejemplo de Gran Bretaña le servía a Keynes para explicar la segunda de las limitaciones, señalando que:

“Gran Bretaña, en los años de preguerra del siglo XX, proporciona un ejemplo de país en que las excesivas facilidades para los préstamos al exterior y la compra de propiedades en el extranjero impidieron a menudo la baja de la tasa de interés que era necesaria para asegurar ocupación plena en el interior" ${ }^{\text {74 }}$.

Finalmente en el caso de la India, su empobrecimiento continuo era consecuencia de un exceso de demanda de dinero no compensado con la entrada de metales preciosos. Las palabras de Keynes eran:

\footnotetext{
${ }^{71}$ Keynes (1980), pp. 297-298.

${ }^{72}$ Keynes (1980), pp. 298-299.

${ }^{73}$ Keynes (1980), p. 299.

${ }^{74}$ Keynes (1980), p. 299.
} 
"La historia de La India en todos los tiempos ha dado el ejemplo de un país de tal manera empobrecido a consecuencia de una preferencia por la liquidez tan apasionada que ni siquiera un influjo enorme y crónico de metales preciosos ha sido suficiente para abatir la tasa de interés a un nivel compatible con el crecimiento de la riqueza real" ${ }^{\text {,75. }}$.

Respecto a la segunda relación que Keynes plantea en el capítulo 23 de su Teoría General, o sea, entre los tipos de interés bajos y la usura, se había manifestado en fechas muy tempranas. Sus ideas sobre los negativos efectos de la usura se pueden entender en una frase de su obra A Tract on Monetary Reform de 1923:

"Los poderes de la usura ininterrumpida son demasiado grandes. Si el monto de los intereses creados creciera sin mitigación por varias generaciones, la mitad de la población sería poco menos que la esclava de la otra mitad"76.

Ya en 1909, preocupado por los efectos de la usura, hizo propuestas para fomentar cooperativas en la India como medio de reducir el coste de los préstamos a los campesinos. Asimismo, en el Simposio organizado en 1932 por The Economic Journal sobre Ahorro y usura (Saving and Usury) ${ }^{77}$, al que asistió Keynes junto a otros destacados economistas como Edwin Cannan, B. P. Adarkar, B.K. Sandwell y K. E. Boulding, el propio Keynes expresó su opinión acerca del pensamiento económico de los doctores pertenecientes a la escolástica tardía ${ }^{78}$. También hizo referencia Keynes en ese Simposio, a los motivos del estancamiento económico en la Edad Media, y entre ellos señalaba que los riesgos eran altos y los ahorros no se canalizaban hacia el capital riesgo, sino que se materializaban en deudas; los tipos de interés eran entonces demasiado altos para que hubiera iniciativas empresariales y se pudiese absorber el ahorro generado. En estas circunstancias, Keynes calificaba a los tipos de interés demasiado altos como de usura ${ }^{79}$.

En su Teoría General, Keynes continúa con ese mismo razonamiento en torno a la usura, y al referirse a los primeros precursores del pensamiento

\footnotetext{
${ }^{75}$ Keynes (1980), p. 299.

${ }^{76}$ En Torrero (1998), p. 876.

${ }^{77}$ Este Simposio fue organizado en respuesta a la publicación del número de diciembre de 1931 de The Economic Journal, que contenía una nota de H. Somerville titulada "Interest and Usury in a New Light”, en la cual sugería que en el Treatise on Money de Keynes se justificaba la actitud de los escolásticos ante el tipo de interés y la usura. Esa nota condujo a ese Simposio sobre "Saving and Usury", plasmado en el siguiente número de The Economic Journal, correspondiente a marzo de 1932, en el que Keynes hizo un resumen de las contribuciones de Somerville, Cannan, Adarkar y Sandwell.

${ }^{78}$ Camacho (2013), p. 589. Para profundizar sobre la opinión de Keynes acerca del pensamiento de los escolásticos pueden consultarse sus Collected Writings, XXIX, The General Theory and Alter. A Supplement, pp.13-16.

${ }^{79}$ Torrero (1998), p. 877.
} 
económico en los siglos XVI y XVII, señalaba que, "había sabiduría en su intensa preocupación por conservar reducida la tasa de interés por medio de leyes de usura" ${ }^{80}$.

De nuevo Keynes, en relación con la usura, hace referencia al caso de la escolástica española en el capítulo 23 de su Teoría General, cuando reconocía que en la formación que él había recibido, la usura se consideraba una propuesta de los escolásticos sin base argumental alguna. En este sentido aclaraba:

"A mí se me hizo creer que la actitud de la iglesia medieval hacia la tasa de interés era intrínsecamente absurda y que los sutiles estudios cuyo objeto era distinguir el rendimiento de los préstamos monetarios del rendimiento de las inversiones activas, eran simples intentos jesuíticos para encontrar una puerta de escape práctica a una teoría necia" ${ }^{81}$.

Sin embargo, Keynes reconocía que la teoría clásica había mezclado de manera confusa la tasa de interés y la eficiencia marginal del capital, conclusión a la que Keynes había llegado tras leer a los escolásticos y considerar que éstos habían hecho "un esfuerzo intelectual honrado" para mantener lo más bajo posible el tipo de interés. La explicación de Keynes sobre esta postura escolástica, quedó reflejada en el siguiente párrafo:

"Porque ahora se ve claramente que las disquisiciones de los eruditos escolásticos tenían por objeto dilucidar una fórmula que permitiera a la curva de la eficiencia marginal del capital ser elevada, mientras aplicaban los reglamentos, las costumbres y la ley moral para conservar baja la tasa de interés",82.

Así entendía Keynes el sentido económico de la prohibición de la usura por parte de los escolásticos, es decir, eliminar o mantener lo más bajo posible los intereses de los préstamos, como mecanismo para impulsar el desarrollo económico ${ }^{83}$. Pocos trabajos reconocen la importancia de las aportaciones escolásticas a la Ciencia Económica y por tanto no han sido suficientemente valoradas por la historiografía internacional ${ }^{84}$ e incluso puede hablarse de un intento de ocultación, como lo demuestra el hecho de que ni en la gran biografía de R. Skidelsky se hace referencia al Simposio de 1932 anteriormente citado, en

\footnotetext{
${ }^{80}$ Keynes (1980), pp. 301-302.

${ }^{81}$ Keynes (1980), p. 311.

${ }^{82}$ Keynes (1980), p. 311.

${ }^{83}$ Sánchez-Lissen (2013), p. 19.

${ }^{84}$ En este sentido debe valorarse positivamente la publicación en 2007, a cargo de Stephen Grabill la obra Sourcebook in Late-Scholastic Monetary Theory. The contributions of Martin de Azpilcueta, Luis de Molina, S.J. and Juan de Mariana, que está dedicada a la memoria de Marjorie GriceHutchinson, "for her pioneering and enduring research in late-scholastic Spanish economic thought”. Incluye colaboraciones de Rodrigo Muñoz de Juana, Francisco Gómez Camacho y Alejandro A. Chafuen. En él se ofrece por primera vez traducido al inglés el trabajo de Martín de Azpilcueta de 1556 Commentary on the resolution of Money, de Luis de Molina Treatise on Money, de 1597 y de Juan de Mariana A Treatise on the alteration of Money, de 1609.
} 
el que Keynes daba su opinión sobre el pensamiento escolástico. Como ha señalado Camacho ${ }^{85}$, con "omisiones como ésta no es extraño que Mark Blaug considere innecesario revisar la historia del pensamiento económico anterior a A. Smith para 'acomodar' la contribución del grupo de Salamanca en la historia del pensamiento económico”.

\section{CONSIDERACIONES FINALES}

A lo largo de este trabajo, nos hemos acercado a la evolución académica y profesional de Keynes a través de seis de sus principales libros que han sido traducidos al castellano, que van desde el primero, Las consecuencias económicas de la paz, aparecido en 1920, hasta la última edición en castellano de su Teoría General en 2014. Cuando llega a Madrid en junio de 1930 era ya un reconocido economista, un persuasivo divulgador a través de la prensa y un ensayista de prestigio. Mostraba también su honda preocupación por los problemas económicos de la Gran Depresión y cómo había que resolverlos, poniendo todavía en esos momentos especial hincapié, más que en la política fiscal, en la aplicación de una política monetaria con bajos tipos de interés que permitiese expandir las economías y aumentar el empleo.

Por otra parte, hemos podido constatar a través de sus libros, las numerosas referencias que Keynes hizo a España, poniendo a nuestro país como ejemplo para explicar las relaciones entre la inflación y la llegada de metales preciosos, especialmente durante el siglo XVI, así como las consecuencias de la acumulación de éstos sobre los salarios y los beneficios empresariales; o la importancia que Keynes dio a los escolásticos españoles de los siglos XVI y XVII, para hacer ver que la usura tenía, en el fondo, un significado económico, como palanca y estímulo de la actividad económica, ideas estas últimas que, sin embargo, no han sido suficientemente reconocidas en la historiografía económica, lo cual ha podido obedecer a ciertos "intereses creados", según la expresión que Keynes utilizó en uno de los últimos párrafos de su Teoría General, al hacer referencia al poder de las ideas de los economistas.

\section{REFERENCIAS BIBLIOGRÁFICAS}

ALMENAR, S. (2001). "La recepción e influencia de Keynes y del keynesianismo en España (1): 1919-1936". En Fuentes Quintana, E. (dir.): Economía y economistas españoles, vol. 6. (pp. 783-851). Barcelona: Galaxia Gutenberg, Círculo de Lectores.

BELTRÁN, L. (1988). "La recepción de Keynes en España". En Rubio de Urquía, R. (coord.): La herencia de Keynes (pp.83-101). Madrid: Alianza.

${ }^{85}$ (2013), pp. 587-591. 
CAMACHO, F. (2013). "Marjorie Grice-Hutchinson". En Sánchez-Lissen, R. (dir. y coord.): Economía y economistas andaluces. Siglos XVI al XX (pp. 587-591). Madrid: Ecobook y Fundación Cajasol.

CLARKE, P. (1988). The Keynesian Revolution in the Making 1924-1936. Oxford: Clarendon Press.

D’ORS, E. (1947). "Keynes”. En Nuevo Glosario, vol. I (p. 186). Madrid: Aguilar.

FERNÁNDEZ PÉREZ, J.M. (1988): Francisco Bernis en la Historia del Pensamiento Económico Español del primer tercio del siglo XX. Madrid: Editorial de la Universidad Complutense.

FUENTES QUINTANA, E. (1981). "El porvenir económico de nuestros nietos. Una conferencia de Keynes en España". Papeles de Economía Española, 6, p. 354.

FUENTES QUINTANA, E. (1983). “John Maynard Keynes en España”. Papeles de Economía Española, 17, pp. 237-334.

FUENTES QUINTANA, E. (1988). "La visión keynesiana de la política económica". En Rubio de Urquía, R.: La herencia de Keynes (pp. 127-149). Madrid: Alianza.

GABRILL, S. (ed.) (2007). Sourcebook in Late-Scholastic Monetary Theory. The contributions of Martin de Azpilcueta, Luis de Molina, S.J. and Juan de Mariana. Lanham: Lexington Books.

GONZÁLEZ GONZÁLEZ, A. (2010). Eugenio D'Ors. El arte y la vida. Madrid: Fondo de Cultura Económica.

HAMILTON, E. (1928). "American Treasure and Andalusian Prices, 1503-1660: A study in the Spanish Price Revolution". Journal of Economic and Business History, (1), pp. 1-35.

HAMILTON, E. (1929a). "American Treasure and the rise of capitalism (1500-1700)". Economica, (27), pp. 338-357.

HAMILTON, E. (1929b). "Imports of American Gold and Silver into Spain, 1503-1600". Quaterly Journal of Economics, vol. 43(3), pp. 436-472.

HAMILTON, E. (1929c). "Wages and Subsistence on Spanish Treasure Ships, 15031660". Journal of Political Economy, vol.37(4), pp. 430-450.

HARROD, R. F. (1951). La vida de John Maynard Keynes. México: Fondo de Cultura Económica. Edición castellana de 1958.

HUERTA DE SOTO, J. (1996). "Lucas Beltrán Flórez: semblanza de un economista". En Beltrán, L.: Ensayos de Economía Política (pp. IX-LXVI). Madrid: Unión Editorial.

KEYNES, J.M. (1919). The Economic Consequences of the peace. Palgrave, Macmillan. KEYNES, J.M. (1923). A Tract on Monetary Reform. London: The Royal Economic Society.

KEYNES, J.M. (1930). A Treatise on Money. London: Macmillan

KEYNES, J.M. (1936). The General Theory of Employment, Interest and Money. London: Macmillan.

KEYNES, J.M. (1971-1989). The Collected Writings, 30 volúmenes. Cambridge University Press: MacMillan.

KEYNES, J.M. (1972). Essays in Persuasion. London: Royal Economic Society.

KEYNES, J.M. (1985). Ensayos sobre intervención y liberalismo. Barcelona: Ediciones Orbis.

NASAR, S. (2013). La gran búsqueda. Barcelona: Debate. 
OLARIAGA, L. (1933). La política monetaria en España. Madrid: Ed. Librería General de Victoriano Suárez.

OLARIAGA, L. (1947). El dinero. Teoría del dinero, vol. I. Madrid: Moneda y Crédito.

OLARIAGA, L. (1953). El dinero. Organización monetaria y bancaria, vol. II. Madrid: Moneda y Crédito.

PABÓN, J. (1969). Cambó. Vol. II. Parte Primera: 1918-1930. Barcelona: Editorial Alpha.

ROJO DUQUE, L.A. (1965). Keynes y el pensamiento macroeconómico actual. Madrid: Tecnos.

ROJO DUQUE, L.A. (1984). John Maynard Keynes: una conmemoración. Discurso de recepción del Académico de Número de la Real Academia de Ciencias Morales y Políticas. Madrid, 6 de noviembre de 1984.

ROJO DUQUE, L.A. (2012). Keynes, su tiempo y el nuestro. Madrid: El Hombre del Tres.

SÁNCHEZ-LISSEN, R. (dir. y coord.). Economía y economistas andaluces. Siglos XVI al $X X$. Madrid: Ecobook y Fundación Cajasol.

SKIDELSKY, R. (2013). John Maynard Keynes. Barcelona: RBA.

TORRERO, A. (1998). La obra de John Maynard Keynes y su visión del mundo financiero. Madrid: Cívitas.

TORRERO, A. (2009). "Keynes (1883-1946). Vida y obra". En Keynes, J.M.: Breve Tratado sobre la reforma monetaria. Escritos (1910-1944). Madrid: Fundación ICO.

TORRERO, A. (2010). "Introducción". En Keynes, J.M. (1930): Tratado sobre el dinero. Madrid: Fundación ICO y editorial Síntesis.

TORRERO, A. (2013). Keynes y la crisis financiera actual. Madrid: Marcial Pons.

UÑA SARTHOU, J. (1920). "Las consecuencias económicas de la paz. Opinión de un economista inglés. Exposición de un libro excepcional". La lectura. Revista de Ciencias y Artes, XX (235), pp. 209-218.

VELARDE, J. (1983). "Biblioteca hispana de Marx, Keynes y Schumpeter. Una primera aproximación". Papeles de Economía Española, (17), pp. 374-416.

VELARDE, J. (1988). "Keynes en España". En Rubio de Urquía, R.: La herencia de Keynes (pp. 102-126). Madrid: Alianza.

VELARDE, J. (1989). "Prólogo". En Olariaga Pujana, L.: Escritos varios (pp. VII-LXXIX). Madrid: Fundación FIES.

VELARDE, J. (2001). "La oleada historicista y el pensamiento económico español". En Fuentes Quintana, E. (dir.): Economía y economistas españoles. Las críticas a la economía clásica (pp. 321-388), vol. 5, Barcelona: Galaxia-Gutenberg, Círculo de Lectores.

VELARDE, J. (2003). "Olariaga y el Banco de España" en Papeles y Memorias de la Real Academia de Ciencias Morales y Políticas, (XI), pp. 77-103. 


\section{ANEXO}

\section{Relación de los libros de Keynes traducidos al castellano por orden cronológico}

1) The economic consequences of the peace (1919), traducido con el título Las consecuencias económicas de la paz. Las ediciones halladas han sido:

- (1920): Traducción de Juan Uña Sarthou, Madrid, Calpe.

- (1987): Traducción de Juan Uña Sarthou y revisión de Lluís Argemí, Barcelona, Crítica. Con una segunda edición en 1991, una tercera en 2002, y una cuarta en 2013.

- (1997): Traducción de Juan Uña Sarthou, Barcelona, Folio.

- (2012): Traducción de Juan Uña Sarthou, Barcelona, RBA.

2) General Theory of Employment, Interest and Money (1936), traducido con el título Teoría General de la ocupación, el interés y el dinero. Las ediciones halladas han sido:

- (1943): Traducción de Eduardo Hornedo, México, Fondo de Cultura Económica. Con otras ediciones y reimpresiones posteriores: 1945, 1951, 1956, 1958, 1963, 1965, 1970, 1971, 1974, 1977, 1980, 1981, 1985, 1986, 1995, 1997, 2000, 2010 y 2014. Las ediciones de 1970 y 1974 llevan una revisión de Ángel Martín Pérez, y la de 2010 una revisión de Roberto Reyes Mazzoni.

- (1993): Traducción de Eduardo Hornedo, Barcelona, Planeta-Agostini.

- (1998): Edición española al cuidado de José Antonio Aguirre, Madrid, Aosta.

- (2002): Traducción de Eduardo Hornedo, Barcelona, RBA. Segunda edición en 2004.

- (2009): Traducción de Juan Carlos Moreno Brid y Rafael Marques Areas. Madrid, Fundación ICO, con Introducción de Antonio Torrero Mañas.

- (2011): Traducción de Eduardo Hornedo, Barcelona, Ciro.

3) Essays in Persuasion (1972), nueva publicación ampliada de la obra de Keynes aparecida en 1931. La edición de 1972 fue publicada originalmente en inglés por Palgrave Macmillan y The Royal Economic Society, y sobre ésta se hizo la traducción al castellano con el título de Ensayos de Persuasión. Las ediciones halladas han sido:

- (1988): Traducción de Jordi Pascual, Barcelona, Crítica.

- (1997): Barcelona, Folio.

- (2009): Traducción de Jordi Pascual, Madrid, Fundación ICO y Editorial Síntesis.

- (2010): Madrid, Ediciones El País.

4) Essays in Biography (1933), traducido con el título Ensayos biográficos: políticos y economistas. La edición hallada ha sido:

- (1992): Barcelona, Crítica.

5) A Tract on Monetary Reform (1923), traducido con el título Breve Tratado sobre la Reforma Monetaria. Las ediciones halladas han sido:

- (1992): México, Fondo de Cultura Económica.

- (2009): Esta edición publicada en Madrid por la Fundación ICO, con una traducción de Carlos Rodríguez Braun cedida por Fondo de Cultura Económica 
(1996), incorpora traducidos por Esther Rabasco, 28 escritos de Keynes aparecidos entre 1910 y 1944, incluidos en The Collected Writings, editados por MacMillan y Cambridge University Press entre 1971 y 1989, con una selección de la Royal Economic Society. Lleva un Prólogo de Aurelio Martínez Estévez y una introducción de Antonio Torrero Mañas.

6) A Treatise on Money (1930), en dos volúmenes: The pure theory of Money (vol. I) y The applied theory of Money (vol. II), traducido con el título Tratado sobre el dinero. La edición hallada ha sido:

- (2010): Traducción de Esther Rabasco. Madrid, Fundación ICO y Editorial Síntesis, con una introducción de Antonio Torrero Mañas. 
